\title{
Das Papsttum und die „vielen“ Italien als Orbis Christianus?
}

\author{
Klaus Herbers
}

\section{Problemstellung}

„Eine unglaubliche Geschichte ist uns zu Ohren gekommen“, so die bewegte Klage eines Papstes im 9. Jahrhundert. In seinem Brief an einen Abt Honorat tadelte er, daß in dessen Kloster der gregorianische Gesang nicht nur abweichend von den Gepflogenheiten des römischen Sitzes, sondern auch von der fast ganzen okzidentalen Kirche, gepflegt werde. Desposco, ich fordere, so fährt der Papst in der ersten Person Singular fort, daß die Gemeinschaft dort nicht länger vom caput, vom Haupt der Kirche abweiche, er droht gegebenenfalls mit Exkommunikation und Anathem, beschwört die Einheit und nennt die römische Kirche mater omnium et magistra vestra ${ }^{1}$.

Das Schreiben ist in zwei Fragmenten der bekannten „Collectio Britannica“ enthalten, die nach den neuesten, noch nicht veröffentlichten Forschungen von Christof Rolker nach 1108 zusammengestellt worden sein soll, und es wird dort einem Papst Leo zugeschrieben, den wir mit Leo IV. (847-855) identifizieren müssen. Ziemlich sicher ist aber der angesprochene Abt nicht, wie früher behauptet, derjenige von Farfa, sondern von S. Salvatore sub monte Lentenano ${ }^{2}$, bei Rieti, an den Grenzen des damaligen Patrimonium Petri, also vor den Toren Roms. Aber warum Ermahnungen in liturgicis; was war mit dem gregorianischen Gesang gemeint? Die musikhistorische Forschung ist uneinig, ob im Kloster beim Monte Lentenano mailändische, süditalische, byzantinisch-griechische oder gar fränkisch bestimmte liturgische Gebräuche vorherrschten, und

1 Vgl. JE 2651, RI 1/4/2/1 Nr. 212; Der Text in Epistolae selectae Sergii II., Leonis IV., Benedicti III., pontificum Romanorum, ed. Adolf von Hirsch-Gereuth, in: MGH Epp. Karol. 3, Berlin 1899, S. 581-614, hier S. 603 f. Nr. 33. Zur Interpretation Klaus Herbers: Leo IV. und das Papsttum in der Mitte des 9. Jahrhunderts. Möglichkeiten und Grenzen päpstlicher Herrschaft in der späten Karolingerzeit, Stuttgart 1996 (Päpste und Papsttum 27), S. 289-295. - Der folgende Text orientiert sich am bei der Tagung gehaltenen Vortrag und bietet nur die wichtigsten Anmerkungen.

2 JE 2651, RI 1/4/2/1 Nr. 212; vgl. auch RI 1/3/1 Nr. 350. 
bleibt entsprechend unsicher, was in diesem Fall unter gregorianisch verstanden wurde ${ }^{3}$.

Die Diskussion um das Fragment, die ich in diesem Einleitungsbeitrag nicht weiter vertiefen möchte, macht für das Anliegen der Tagung und des hier vorgelegten Bandes mehrerlei deutlich:

- ein Papst des 9. Jahrhunderts greift mit römischer Autorität an den Grenzen des Patrimonium Petri zugunsten einheitlicher liturgischer Gebräuche ein,

- $\quad$ die fehlende Einheit wird - so jedenfalls die Vermutungen der Forschung auf sehr verschiedene, in Italien konkurrierende Traditionen zurückgeführt,

- das Schreiben postuliert eine Einheitlichkeit der okzidentalen Kirche, von der man am Monte Lentenano abweiche ${ }^{4}$,

- und schließlich: Das Fragment ist zwar seit der Entdeckung der „Collectio Britannica" 1877/78 bekannt, aber wegen der zweifelhaften Zuordnung nicht in die Italia Pontificia eingegangen, wo es in Band 4 auf Seite 25 eingeschoben werden müßte.

Wir wissen nicht, ob Leo IV. langfristig bezüglich Liturgie und Gesang am Monte Lentenano Erfolg hatte. Wie auch immer: Der Text erschien offensichtlich an der Wende zum 12. Jahrhundert wichtig genug, um ihn in eine kanonistische Zwischensammlung aufzunehmen. Dazu tritt ein weiterer Aspekt: Leo IV. wollte offensichtlich - und der Kompilator unterstrich dies durch seine Auswahl - vereinheitlichend und integrierend wirken. War dies eine Ausnahme oder die Regel?

Mein Beitrag, der einige Perspektiven des Bandes eröffnend ansprechen soll, kann sich nicht darauf beschränken, die den meisten bekannte Vielfalt Italiens im frühen und hohen Mittelalter nur einfach in Erinnerung zu rufen. Vielmehr will ich ausgehend von dem skizzierten Beispiel danach fragen, inwieweit Aktionen und Reaktionen der Päpste in einem Prozeß des Mit- und Nebeneinanders dieser Vielfalt integrierend und/oder desintegrierend beabsichtigt waren oder wirkten. Dies könnte neue Interpretationsmöglichkeiten bieten, weil herkömmliche Thesen zur Geschichte des Papsttums im frühen und hohen Mittelalter davon ausgehen, daß die Päpste vor allem seit dem 11. Jahrhundert zu einer lateinischen Einheit des westlichen Europa maßgeblich beigeträgen hätten. Diesen Überlegungen stehen neuere Interpretationen entgegen, die eher umgekehrt die Vielfalt als das Kennzeichen auch des mittelalterlichen Europa

3 Vgl. hierzu Herbers: Leo (wie Anm. 1) S. 292, mit Referat der teilweise kontroversen Forschungen.

4 Epistolae selectae (wie Anm. 1) S. 604 Nr. 33: ... ab omni pene occidentali aecclesia, et prorsus ab omnibus, qui Latinis vocibus laudem aeterno regi conferunt et sonos canoros persolvunt, dissentiatis. 
postulieren ${ }^{5}$ und damit indirekt auch die Integrationsleistungen des hochmittelalterlichen Papsttums relativieren. Daraus ergibt sich die Frage, ob und inwieweit die Integrationskraft des Papsttums vor den Toren Roms, in Italien, besonders gut wirksam werden konnte. War Italien so etwas wie ein Orbis christianus, en miniature" oder hatten die Päpste vielleicht gerade hier besondere Schwierigkeiten zu gewärtigen, weil die Vielfalt dem entgegenstand? Wie weit reicht die These, daß der Papst in dieser Zeit in Italien - anders als bei anderen Empfängergruppen - weniger als "Stellvertreter des Apostelfürsten gesehen wurde, sondern als Mitbischof in Italien“, die Jochen Johrendt für das 10. und beginnende 11. Jahrhundert vertreten hat ${ }^{6}$, über diesen Zeitraum hinaus?

Der vorgelegte Band berücksichtigt damit, zweitens, die Voraussetzungen der Arbeiten von Paul-Fridolin Kehr und seiner Mitarbeiter, der Italien pragmatisch vor dem Hintergrund einer sich stets verändernden Karte der ApenninHalbinsel in eine Italia Media (später Band 1-4), eine Italia superior (Band $5-7$ ) und eine Italia inferior (Band 8-10) unterschied ${ }^{7}$. Die Tagung war aber zugleich - angesichts des weitgehenden Abschlusses der Italia Pontificia ${ }^{8}-$ dem $^{2}$ Grundsatz verpflichtet, die Sammlung der Papsturkunden als die Voraussetzung für tiefergehende Interpretationen zu nutzen ${ }^{9}$ und die deutende Untersuchung des Materials nicht nur anderen zu überlassen. Dies entspricht im übrigen Kehrs eigener Meinung, der immer wieder bedauerte, daß die Fülle der Arbeit die Zeit zur Interpretation des Materials kaum zulasse, aber trotzdem vielfältig Stellung nahm, wie der 2005 von Rudolf Hiestand besorgte Nachdruck der Ausgewählten Schriften Kehrs eindrücklich belegt ${ }^{10}$.

5 Michael Borgolte: Europa entdeckt seine Vielfalt 1050-1250, Stuttgart 2002 (Handbuch der Geschichte Europas 3), zu verschiedenen Europakonzeptionen vgl. jetzt auch Klaus Herbers: Europa und seine Grenzen im Mittelalter, in: Grenzräume und Grenzüberschreitungen im Vergleich. Der Osten und der Westen des mittelalterlichen Lateineuropa, hg.v. Klaus Herbers/Nikolas Jaspert, Berlin 2007 (Europa im Mittelalter. Abhandlungen und Beiträge zur historischen Komparatistik 7), S. 21-41.

6 Jochen Johrendt: Papsttum und Landeskirchen im Spiegel der päpstlichen Urkunden (896-1046), Hannover 2004 (MGH Studien und Texte 33), zusammenfassend S. 261.

7 Vgl. Rudolf Hiestand: Die Italia Pontifica, in: Das Deutsche Historische Institut in Rom 1888-1988, hg.v. Reinhard Elze/Arnold Esch, Tübingen 1990 (Bibliothek des Deutschen Historischen Instituts 70), S. 167-189, hier S. 177.

8 Rudolf Hiestand: Die unvollendete Italia Pontificia, in: Hundert Jahre Papsturkundenforschung. Bilanz - Methoden - Perspektiven. Akten eines Kolloquiums zum hundertjährigen Bestehen der Regesta Pontificum Romanorum vom 9.-11. Oktober 1996 in Göttingen, hg.v. DEMs., Göttingen 2003 (AAG, phil.-hist. Kl., 3. Folge 261), S. 47-57.

9 Vgl. zum Beispiel Paul Fridolin KeHR: Zur Geschichte Wiberts von Ravenna (Clemens III), in: SPA. PH, Berlin 1921, H. 19, S. 355-368 (Nachdr. in: DERs.: Ausgewählte Schriften, 2 Bde, hg.v. Rudolf Hiestand, Göttingen 2005 [AAG, phil.-hist. Kl., 3. Folge 261], Bd. 1 S. 209-222, hier S. 209).

10 KeHr: Schriften (wie Anm. 9). 
Die Italia ist aber unvollendet ${ }^{11}$. Deshalb weise ich schließlich auch auf einige Quellengruppen hin, die der Diplomatiker Kehr, „der auf Urkunden pürschte", weniger im Blick hatte, denn die zitierte These von Jochen Johrendt hat dieser vor allem durch eine Untersuchung der von Harald Zimmermann vorgelegten Urkunden für das 10. und beginnende 11. Jahrhundert (8961046) gewonnen ${ }^{12}$.

Diese drei Perspektiven verdeutliche ich mit fünf kurzen Aperçus, die zeitlich und räumlich zumindest eine gewisse Varianz aufweisen sollen, aber von eigenen Arbeitsschwerpunkten mitbestimmt sind. Dies dürfte deshalb kein grundsätzlicher Nachteil werden, weil die Beiträge des Bandes dies erweitern und weil es zunächst vor allem auf die Fragestellungen ankommt.

\section{Italien und Rom - Objekte der Begierde oder Hinterland?}

Die Bezeichnung Italien - die auch von Kehr benutzt wurde - leitet fehl, denn die Geschichte Italiens ist nicht einfach eine Fortsetzung der altrömischen, obwohl dies Vertreter der Renaissance wiederholt behaupteten, und die Publizistik des Risorgimento dies weiter unterstrich. Natürlich gab es die Bezeichnung Italia schon seit der Spätantike, vor allem als geographische Bezeichnung für die Apenninen-Halbinsel. Als diese Halbinsel durch den römischen Stadtstaat in der Antike politisch geeint wurde, verstand man unter Italien das Gebiet südlich von Magra und Rubikon, die Po-Ebene galt jedoch als Gallia Cisalpina, die erst Caesar Italien angliederte.

Ganz anders war der Sprachgebrauch einige Jahrhunderte später. Zwar verwendete man weiterhin den geographischen Begriff, aber nach dem Untergang des Langobardenreiches (568-774), das seine Schwerpunkte im wesentlichen in der Po-Ebene und der Toskana besaß, kam für dieses Areal die Bezeichnung Regnum Italiae auf, viele hochmittelalterliche Quellen meinen mit Italia entsprechend oft eher den nördlichen Teil der Halbinsel. Die Mitte und der Süden standen nach den oströmischen Eroberungen und den Gotenkriegen des 6. Jahrhunderts teilweise wieder unter oströmischer bzw. byzantinischer Herrschaft, so daß die Namen Rom und Neu-Rom zeitweise wichtiger als die Bezeichnung Italien wurde. Da aber neben den verschiedenen teilweise unabhängigen Herrschaften sowohl mit dem Regnum Italiae als auch mit den byzantinisch dominierten Gebieten eigentlich Territorien mit Leuten existierten, die Herrschern in fernen Zentren unterstanden, wurde das Schicksal Italiens

11 Vgl. den Titel der in Anm. 8 genannten Abhandlung von Rudolf Hiestand.

12 Harald Zimmermann: Papsturkunden 896-1046, 3 Bde., Wien 1988-1989 (Österreichische Akademie der Wissenschaften, phil.-hist. Kl. Denkschriften 174, 177, 198) (Bd. 1 und 2 in 2. Aufl.). 
vielfach nicht auf der Apenninen-Halbinsel, sondern anderswo entschieden. Italien oder Teile davon waren eventuell das ferne Hinterland, wurden aber in bestimmten Situationen zum Zankapfel und Objekt der Begierde und des Streites, der durch kirchliche Gliederung der Einflußbereiche der Patriarchen von Konstantinopel und Rom weiter verschärft werden konnte.

Verfolgt man die Entwicklung der Karte im Laufe des Mittelalters weiter, so blieb neben und innerhalb des Regnum Italiae eine Vielzahl kleiner und fast eigenständiger Herrschaften besonders an den Küsten bestehen. Weiterhin gab es vor allem im ravennatischen Raum und in Süditalien einen starken byzantinischen Einfluß, der nur teilweise die fast unabhängigen kleinen Herrschaften wie Neapel, Gaeta oder Amalfi integrieren konnte. Hinzu traten ab dem 9. Jahrhundert muslimische Territorien in Süditalien, besonders in Sizilien sowie schließlich die entstehenden Normannenreiche im 11. und 12. Jahrhundert. Im Zentrum lagen aber Rom und das Patrimonium Petri, wo die Päpste zunächst mit starker Rückbindung an den oströmischen Kaiser Einfluß besaßen, seit dem 8. Jahrhundert aber mit Legitimation der Karolinger und der neuen westlichen Kaiser weitgehend selbständig agierten; deshalb sei dieser Bereich hier ausgespart.

Wo also beginnen, und wo enden Rom und Italien? Gehören die Kontakte mit den oströmischen Kaisern, soweit sie Rom betreffen, zur Italia Pontificia? Wie ist zu verfahren, wenn oströmischer Kaiser und Patriarch von Konstantinopel die Kontrahenten Roms sind? Werden dann Kaiser und Patriarch getrennt, einmal in IP 11 („Reges et Imperatores“) und einmal in einen Oriens graecus pontificius? Die Beiträge von Matthias Maser und Guglielmo Cavallo setzen sich mit diesem komplizierten Geflecht näher auseinander ${ }^{13}$; einige Problempunkte thematisiert mein zweites Aperçu:

\section{Süditalien - ein kleines, vielfältiges Europa?}

Byzanz trat nicht nur immer wieder in direkten Kontakt mit Rom und anderen Zentren Italiens. Besonders die Position Siziliens änderte sich im 8. und 9. Jahrhundert. Verweist die Abschrift des Registers von Papst Gregor I. noch häufig auf Sizilien und die dortigen päpstlichen Patrimonien, wie die chronologische Übersicht in IP 10 gut verdeutlicht ${ }^{14}$, so änderte sich dies unter dem

13 Siehe unten, S. 39-68 und 69-83.

14 Gregor der Große: Registrum epistularum, ed. Dag Norberg, Turnhout 1982 (CCL 140, 140A), vgl. auch (vor allem des Kommentars wegen immer noch) Gregor der Große: Registrum epistularum, ed. Paul Ewald/Ludo Moritz Hartmann, Berlin 1891 1899 (MGH Epp. 1-2); vgl. auch den Elenchus vor allem in IP 10, S. XIX-XXVIII. 
Einfluß byzantinischer Interessen und noch mehr nach der weitgehenden Inbesitznahme der Insel durch die Muslime im 9. Jahrhundert.

Seitdem die kirchlichen Institutionen Siziliens und Kalabriens während des Bilderstreites von Kaiser Konstantin V. (741-775) gezwungen worden waren, sich dem Patriarchat von Konstantinopel zu unterstellen ${ }^{15}$, war nach den vielen frühmittelalterlichen ein weiterer Konflikt mit den römischen Päpsten, die dies nicht anerkannten, vorprogrammiert. Sizilien aber wurde für den Patriarchat von Konstantinopel kurzzeitig zur Kaderschmiede: Patriarch Methodios (843847) stammte von dort, und Erzbischof Gregor Asbestas von Syrakus (845863) wäre auch fast zum Patriarchen in Konstantinopel aufgestiegen, unterlag aber Ignatios. Der Eklat folgte: Bei der Weihe des Ignatios zum Patriarchen soll der hitzige Gregor von Syrakus die Kerzen, die er in Händen hielt, zu Boden geworfen haben ${ }^{16}$, wurde von Ignatios der Kirche verwiesen und soll den neuen Patriarchen sodann verwünscht haben. Gegen seine darauf folgende Absetzung (847/48) appellierte er aber an den römischen Stuhl und bot damit dem Papst eine Steilvorlage, jurisdiktionelle Ansprüche gegenüber dem Patriarchen von Konstantinopel zu formulieren ${ }^{17}$. Sizilien wurde so teilweise zum Schauplatz für einen Ost-West-Konflikt, war mit dem übrigen Süditalien immer wieder das Mittelglied einer Dreiecksgeschichte, die kaum aus der Perspektive der Italia Pontificia allein zu erfassen ist. Beachtenswert ist auch hier wiederum die Überlieferung: Nicht Urkunden, sondern Registerfragmente, Konzilsakten sowie Parteischriften und historiographische Quellen bieten die wichtigsten Informationen ${ }^{18}$.

Süditalien erscheint in diesem Falle somit eher als Austragungsort oder als Schlachtfeld für andere Beziehungen oder Konflikte. Was sich aber an Mög-

15 Die Datierung ist umstritten, vgl. Herbers: Leo (wie Anm. 1) S. 302, zu verschiedenen Vorschlägen der Forschung.

16 Vgl. z. B. Anastasius Bibliothecarius in seiner Praefatio zur lateinischen Fassung des 8. ökumenischen Konzils, ed. Anastasii bibliothecarii epistolae sive praefationes, ed. Ernst Perels/Gerhard Laehr, in: MGH Epp. Karol. 5, Berlin 1928, S. 395-442, hier S. 404 Nr. 5; vgl. die Vita Ignatii, ed. Mansi 16 Sp. 232; MPG CV Sp. 512. Demnach warf Gregor die Kerzen, die er in der Hand hielt, zu Boden. Dies könnte bewußt auf einen Exkommunikationsritus anspielen. Vgl. die weiteren Nachweise bei Venance Grumes/ Jean Darrouzìs: Les regestes des actes du Patriarcat de Constantinople 1/2: Regestes de 715 à 1043; 1/3: Regestes de 1043 à 1206. 2. Aufl. von Jean Darrouzès, Paris 1989 (Le partriarcat Byzantin, Série 1) Nr. 445. Zum Hintergrund Patricia Karlin-Hayter: Gregory of Syracuse, Ignatios and Photios, in: Iconoclasm. Papers Given at the Ninth Spring Symposium of Byzantine Studies, University of Birmingham March 1975, hg.v. Anthony Bryer/Judith Herrin, Birmingham 1977, S. 141-145 und Herbers: Leo (wie Anm. 1) S. 303.

17 Ebd. S. $304 \mathrm{f}$.

18 Vgl. zum Beispiel die einschlägigen Nummern der Regesten von Grumel/Darrouzès (wie Anm. 16), sowie bei RI 1/4/2/1. 
lichkeiten anbot, um in diesem Raum auch päpstlichen Einfluß stärker zur Geltung zu bringen und vereinheitlichend $\mathrm{zu}$ wirken, sollte schon deshalb wieder problematisch werden, weil gleichzeitig arabisch-berberisch-muslimische Bedrohungen neue Machtkonstellationen erzwangen. Die sarazenischen Schiffe, teilweise Freibeuter, teilweise aus dem aghlabidischen Nordafrika kommend, suchten sogar 846 Rom heim ${ }^{19}$ und verunsicherten noch das ganze 9. Jahrhundert hindurch die Küsten des Patrimonium Petri. Die Päpste reagierten und mußten nun mit den süditalischen civitates, mit Byzanz und den karolingischfränkischen Herrschern den Schulterschluß suchen ${ }^{20}$.

Papst Johannes VIII. (872-882) bemühte sich z. B. um ein byzantinisches Schutzkontingent von zehn Kriegsschiffen (Chelandien) ${ }^{21}$. Der Papst ermahnte die süditalischen civitates immer wieder, ihre Bündnisse mit den Sarazenen aufzugeben, jedoch ohne langfristigen Erfolg. In Traetto, im Süden des Patrimonium Petri, handelte er mit einigen süditalischen Stadtstaaten 877 nur die Grundzüge einer Abmachung aus. Ohne ,Schutzgelder' war kaum Ruhe herzustellen. Amalfi sollte die Küsten des Patrimonium Petri gegen eine Summe von 10.000 Mankusen schützen ${ }^{22}$. Die Amalfitaner versuchten jedoch ${ }^{23}, 12.000$ Mankusen herauszupressen. In dieser unsicheren Situation brach Johannes 878 mit dem Schiff ins Frankenreich auf ${ }^{24}$. Zuvor schloß er ein Abkommen mit den Sarazenen ${ }^{25}$ : Für 25.000 Mankusen wollte er Sicherheit erkaufen. „Der Stuhl Petri zinste den Anhängern des Lügenpropheten, damit sie sein Gebiet nicht völlig zu Grunde richteten und zur Einöde machten. Dahin führte der Mangel

19 IP 10 S. 467 Nr. 13, RI 1/4/2/1 Nr. 59 und 60.

20 Vgl. allgemein hierzu Nicola Cilento: I Saraceni nell'Italia meridionale nei secoli IX e X, in: Archivio storico per le province Napoletane, N. S. 38 (1959) S. 109-122, sowie die folgenden Anm.

21 JE 3092, ed. Registrum Johannis VIII. papae, ed. Erich CAspar, in: MGH Epp. Karol. 5, Berlin 1928, S. 1-272, hier S. 45 Nr. 47. Vgl. später JE 3303, ed. Registrum Johannis VIII., S. 214 Nr. 245.

22 Schutzvertrag: JE 3126, ed. Registrum Johannis VIII. (wie Anm. 21) S. 75 Nr. 79. Die Mankusen scheinen hier offensichtlich als Rechnungseinheit zu dienen.

23 Dies geschah unter falscher Aussage gegenüber früheren Abmachungen, wie Johannes in einem Brief an Salerno kommentiert: JE 3127, ed. Registrum Johannis VIII. (wie Anm. 21) S. 81 Nr. 86.

24 Johannes FrIED: Boso von Vienne oder Ludwig der Stammler. Der Kaiserkandidat Johannes VIII. in: DA 32 (1976) S. 193-208; vgl. weiterhin Hubert Mordek/Gerhard Schmitz: Papst Johannes VIII. und das Konzil von Troyes (878), in: Geschichtsschreibung und geistiges Leben im Mittelalter. Festschrift für Heinz Löwe zum 65. Geburtstag, hg.v. Karl Hauck/Hubert Mordek, Köln 1978, S. 179-225, bes. S. $179 \mathrm{f}$.

25 JE 3139, ed. Registrum Johannis VIII. (wie Anm. 21) S. 85 Nr. 89, RI 1/3/1 Nr. 551; Fred Engreen: Pope John the Eighth and the Arabs, in: Speculum 20 (1945) S. $318-$ 330, hier S. 321 Anm. 4. 
eines mächtigen Kaisers und Schirmherrn der Kirche", so kommentierte noch Ernst Dümmler dieses Ereignis ${ }^{26}$.

Dies war ein Tribut, kein Vertrag wie ihn hingegen einige süditalische $c i$ vitates mit den Sarazenen geschlossen hatten: Gegen diese pacta oder foedera impia der Süditaler, zog Johannes VIII. in mehr als zwanzig meist in seinem Register erhaltenen Briefen zu Felde ${ }^{27}$. Er selbst schloß 878 mit den Sarazenen keinen Vertrag, sondern leistete Tribut. Vielleicht war sogar das Geld in dieser direkten Zahlung besser als bei den Amalfitanern oder anderen Küstenstädten angelegt, denn auch nachdem Johannes nach Rom zurückgekehrt war, blieben weitere Verhandlungen mit den süditalischen civitates schwierig. So versprach der Papst beispielsweise Bischof Athanasius von Neapel eine Geldsumme, damit dieser seine sarazenischen Freibeuter entlasse. Dieser schickte jedoch nur die bisherigen Helfer heim, ließ sich das Geld auszahlen, um anschließend neue, in Sizilien rekrutierte Sarazenen einzustellen ${ }^{28}$.

Ein Versuch, im Dezember 879 den Schutz für die Küsten Roms von den Amalfitanern zu erkaufen ${ }^{29}$, verdeutlicht die komplizierte Situation. Johannes VIII. versprach ihnen über die schon 877 vereinbarten 10.000 Mankusen hinaus weitere 1.000 sowie Zollfreiheit im römischen Hafen. Sollten sie aber das Bündnis mit den Sarazenen nicht lösen, drohte der Papst mit einer Handelssperre $^{30} .886$ dachte Papst Stephan V. daran, den Handel mit Neapel gegebenenfalls ernstlich zu behindern ${ }^{31}$. Die Versuche zur Errichtung einer gemeinsamen Front und die faktisch desintegrativen Kräfte dieser Konstellation sind offensichtlich. In der Überlieferung ist es aber wieder weniger urkundliches als briefliches und kanonistisches Material, das dank der Registerabschrift zu Jo-

26 Ernst Dümmler: Geschichte des Ostfränkischen Reiches, 3 Bde., Leipzig, 2. Aufl. $1887-1888$ (Nachdr. 1960), Bd. 3 S. 73.

27 Vgl. Giorgio Balladore/ Giulio Vismara: Acta Pontificia Juris Gentium usque ad annum MCCCIV, Mailand 1946, S. 270-274, Nr. 37-63.

28 JE 3343, ed. Registrum Johannis VIII. (wie Anm. 21) S. 241 Nr. 273, und JE 3346, ed. Registrum Johannis VIII., S. 246 Nr. 279; vgl. Ekkehard EıскноғF: Seekrieg und Seepolitik zwischen Islam und Abendland. Das Mittelmeer unter byzantinischer und arabischer Hegemonie (650-1040), Berlin 1966, S. 230. Vgl. auch Erchembert, Historia Langobardorum Beneventanorum, ed. Georg WaITz, in: MGH SRL 1, Hannover 1878, S. $231-264$, hier S. 255.

29 JE 3308, ed. Registrum Johannis VIII. (wie Anm. 21) S. 218 f. Nr. 250; vgl. zu früheren Anstrengungen im September z. B. JE 3278, ed. Registrum Johannis VIII., 192 Nr. 214.

30 Ebd.

31 JL 3414, ed. Fragmenta registri Stephani V. papae, ed. Erich CAspar, in: MGH Epp. Karol. 5, Berlin 1928, S. 334-353, hier S. 337 Nr. 7: Et non dicas, quia, si domnus apostolicus veniens messes nostras deleverit, habemus alias provincias, unde labores habere possimus. Nam nos et Romam Sardiniam Corsicam et totam christianitatem contra te claudemus, ut nullo modo recuperare valeatis. 
hannes VIII. umfassend befragt werden kann, über Empfängerarchive jedoch wenig aussagt.

Gab es aber wirklich keine direkten Kontakte mit den neuen muslimischen Machthabern auf der Insel Sizilien, teilweise auch an anderen Orten Süditaliens? Die Suche in der IP 10 bietet zumindest ein Stück zu Papst Marinus I., der den Emir von Sizilien gebeten habe, daß er den Erzbischof (von Syrakus) und den Bischof von Mileto freilasse und die von den Sarazenen gefangenen Sklaven ausliefere. Indes, das Stück ist erst im 18. Jahrhundert in sizilianischer Sprache überliefert, und Dieter Girgensohn hat mit Recht ein Fälschungskreuz vor das entsprechende Regest gestellt ${ }^{32}$.

Vor dem Hintergrund dieses Beleges habe ich vor einigen Jahren noch weitere Bemerkungen zu päpstlichen Aktivitäten gesucht und gefunden, die bisher in die gängigen Verzeichnisse zur Papstgeschichte von Jaffé und in die Italia Pontificia nicht eingegangen sind. Aber hier ist die Überlieferung problematisch: Sie sind angeblich in arabischer Sprache aufgezeichnet, wurden spätestens im 17. Jahrhundert ins Sizilianische übersetzt und in die Jahre 883 bis 887 datiert, damit den Päpsten Marinus I., Hadrian III. und Stephan V. bzw. Al-Hasan ben al Abbas, dem Emir von Syrakus, zugeordnet. Es geht um den Freikauf von Sklaven. Die Muslime, so heißt es in dem letzten dieser einschlägigen Briefe, seien besser als die Christen, denn sie würden Sklaven für Geld freilassen. Dem päpstlichen Wunsch, künftig von Versklavungen ganz abzusehen, könne jedoch nicht entsprochen werden; die Griechen seien ja ebenso gefürchtete Sklavenjäger. Nach verschiedenen Prüfungen steht inzwischen soviel fest: In diesen Schreiben wurden wohl erst von sehr späten Autoren angebliche Nachrichten über die sarazenische Gefangenschaft des Bischofs von Syrakus und anderer Sizilianer zu einem fiktiven Briefwechsel verarbeitet ${ }^{33}$. Dabei haben diese sogar angeblich arabische „Urfassungen“ aufgezeichnet, deren Schriftbild zwar fremde Zeichen suggeriert, die mit dem Arabischen aber nichts zu tun haben. Diese literarischen Abenteuer des 18. Jahrhunderts möchte ich noch weiter verfolgen ${ }^{34}$, für die Italia Pontificia werden auch diese Schreiben im

32 IP 10 S. 186 Nr. $† 72$.

33 In diesen Zusammenhang könnte auch das von Girgensohn in Anm. 32 erwähnte Stück, das Marinus I. zugeschrieben wird, gehören.

34 Vgl. die (phantasiehaften) Abb. und Übertragungen bei Alfonso Arroldi (ed.): Codice diplomatico Arabo-Siciliano, Palermo 1789, für die hier interessierende Zeit S. 244263. Diese Aufzeichnungen wurden vor einigen Jahren noch als echt angesehen von Raffaele Tucciarone: I saraceni nel ducato di Gaeta e nell'Italia centromeridionale (secoli IX e X), Gaeta 1991, bes. S. 87-99; vgl. jedoch schon den Eintrag von Guiseppe Gabrieli: Airoldi, Alfonso in der Enciclopedia Italiana di scienze, lettere ed arti 2, Mailand 1929, S. 36, der unterstreicht, daß Airoldi zunächst den Fälschungen von Giuseppe Vella Glauben schenkte. Zu Giuseppa Vellas Wirken vgl. die Monographie von Thomas Freller: The Rise and Fall of Abate Giuseppe Vella. A story of forgery and 
Nachtragsband - ähnlich wie Kehr bei den gelehrten Pisaner Erfindungen ${ }^{35}$ mit einem Fälschungskreuz zu versehen sein. Dennoch läßt sich nicht leugnen, daß die Texte die Situation des 9. Jahrhunderts, als sich Muslime, Griechen sowie Venezianer rege am Sklavenhandel beteiligten, teilweise treffen könnten. Dafür gibt es weiterhin zumindest einen Anknüpfungspunkt, in der zeitgleichen Vita Stephans V. (885-891): Bei Beginn seines Pontifikates sei alles in Rom geplündert und leer gewesen, der neue Papst habe über nichts verfügt, um Klerus und Scholen zu beschenken oder um Gefangene zurückzukaufen ${ }^{36}$. Weniger die Kontakte mit den Arabern als vielmehr das Schicksal der Gefangenen scheint zentral gewesen zu sein. Gefangene auszulösen war in Rom nichts Außergewöhnliches, kein Novum. Mehrere Briefe Gregors I. von 592 bis 598 berichten davon ${ }^{37}$, auch Johannes IV. griff um 640 in den dalmatinischen Gefangenenhandel ein ${ }^{38}$. Johannes VIII. ermahnte sardische Fürsten, die von Griechen gekauften heidnischen Sklaven freizulassen ${ }^{39}$, obwohl die päpstliche Sorge meist nur christlichen Sklaven galt. Für die Päpste war aber das Problem des Gefangenenfreikaufes vor allem im früheren Mittelalter in Italien nicht erst seit den Sarazenenzügen auch in praktischer Hinsicht akut. Damit bot aber die italische Perspektive dieser Epoche auch Orientierungen für juristische Festlegungen und weiteres Verhalten in der Kreuzzugszeit.

\section{Konkurrenz oder Einheit?}

Obwohl die neuen Konstellationen im Süden ausreichend Konfliktstoff boten, versuchten die Päpste ebenso im Norden, beispielsweise in Ravenna, eigenständige Tendenzen einzudämmen oder bei Streitigkeiten zwischen Aquileja und Grado, die auch in der unterschiedlichen Orientierung hinsichtlich Byzanz mit

deceit, Malta 2001 (ich danke Herrn Kollegen Titus Heydenreich in Erlangen und Herrn Dr. Thomas Freller für ihre Informationen).

35 Vgl. hierzu IP 3 S. 355 ff. sowie Paul Fridolin KeHR: Der angebliche Brief Paschals II. an die Consuln von Pisa und andere Pisaner Fälschungen, in: QFIAB 6 (1904) S. 316342.

36 ... vel unde captivos redimeret..., „Liber pontificalis“, ed. Louis Duchesne: Le Liber pontificalis. Texte, introduction et commentaire, 2 Bde., Bd. 3 hg.v. Cyrille Vogel, Paris 1886-1892, 1957, hier Bd. 2 S.192.

37 Gregor: Register (wie Anm. 14); JE 1210, 1361, 1412, 1459, 1469, 1510, vgl. Acta Pontificia juris gent. (wie Anm. 27) S. 446 Nr. 36-41.

38 JL Bd. 1 S. 228.

39 JE 2983, ed. Fragmenta registri Johannis VIII. papae, ed. Erich CAspar, in : MGH Epp. Karol. 5, Berlin 1928, S. 273-312, hier S. 288 f. Nr. 27; IP 10 S. 379 Nr. 26. - Vgl. auch den Bericht über den Freikauf des Erzbischof Rotland von Arles, für den die Sarazenen Lösegeld kassiert haben sollen, obwohl er bereits gestorben war, vgl. den bewegenden Bericht in den Annales Bertiniani, ed. Felix Grat/Jeanne Vielliard/Suzanne Clémencet: Les Annales de Saint-Bertin, Paris 1964, ad a. 869, S. 165 f. 
begründet waren und bis nach Venedig hin ihre Auswirkungen hatten, zu vermitteln ${ }^{40}$. Die Beiträge von Antonio Carile und Maria Pia Alberzoni behandelten auf der römischen Tagung die wichtigen eigenständigen Zentren Ravenna und Mailand ${ }^{41}$.

Manche dieser Zentren stellten auch Rom mit Traditionen und Heiligenkulten einen Ausweis ihrer Eigenständigkeit entgegen. Apostelkulte waren besonders wirksam. Dies ließe sich am Beispiel Venedigs und des Markuskultes sehr gut zeigen ${ }^{42}$. Noch deutlicher wird am Beispiel Benevents, worüber JeanMarie Martin handelt, wie die skizzierten neuen Konstellationen Süditaliens veränderte politische und kirchenpolitische Schwerpunkte begünstigten. Das Vordringen und die Eroberungen der Aglabiden und weiterer muslimischer Gruppen in Süd- und Mittelitalien ${ }^{43}$ förderte - glaubt man den Berichten - eine verstärkte Übertragung von Reliquien. Besonders interessant ist der Fall des Apostels Bartholomäus. Seine Gebeine wurden 838 von Lipari (wohin sie 580 gekommen sein sollen) nach Benevent übertragen ${ }^{44}$. Was wird im zugehörigen

40 Dies hat Paul Fridolin Kehr unter anderem in der grundlegenden Studie zu Rom und Venedig deutlich gemacht, vgl. Paul Fridolin KeHR: Rom und Venedig bis ins 12. Jahrhundert, in: QFIAB 19 (1927) S. 1-180.

41 Zum Beitrag von Maria Pia Alberzoni siehe unten S. 135-181. Der Beitrag von Antonio Carile fehlt in diesem Band.

42 Alfons ZetTler: Die politischen Dimensionen des Markuskults im hochmittelalterlichen Venedig, in: Politik und Heiligenverehrung im Hochmittelalter, hg.v. Jürgen Petersohn, Sigmaringen 1994 (VuF 42), S. 541-571.

43 Grundlegend zum muslimischen Italien: Michele Amari: Storia dei musulmani di Sicilia, 3 Bde., Florenz 2002-2003 (= Neuausgabe mit Angabe der Originalpaginierung der Ausgabe von 1837-1839) (zur fraglichen Zeit bes. Bd. 1 und 2); Rudolf Hiestand: Byzanz und das Regnum Italicum im 10. Jahrhundert, Zürich 1964 (Geist und Werk der Zeiten 9), bes. S. 19-32; Eickhoff: Seekrieg (wie Anm. 28); Horst Enzensberger: Unteritalien seit 774, in: Handbuch der Europäischen Geschichte, Bd. 1, hg. v. Theodor Schieffer, Stuttgart 1976, S. 784-804. Zum Beitrag Jean-Marie Martin siehe unten S. $109-133$.

44 Vgl. hierzu Anastasius Bibliothecarius, Sermo Theodori Studitae de sancto Bartholomeo apostolo, hg.v. Ulla Westerbergh, Stockholm 1963 (Acta Universitatis Stockholmiensis. Studia Latina Stockholmiensia 9), vor allem S. 10-17, und allgemein Antonio Vuolo: „Agiografia Beneventana“, in: Longobardia e longobardi nell'Italia meridionale. Le istitutizioni ecclesiastiche, hg.v. Giancarlo Andenna/Giorgio Picasso. Atti del $2^{\circ}$ convegno internazionale di studi promosso dal Centro di Cultura dell'Università cattolica del Sacro Cuore. Benevento 29-31 maggio 1992, Mailand 1996, S. 199-237. Einige Aspekte des Bartholomäuskultes hat Kerstin STEINIGER: Bartholomäusverehrung in Benevent vor dem Hintergrund der politischen Situation in Süditalien im 9. und 10. Jahrhundert (unveröff. Zulassungsarbeit Erlangen 2000) erarbeitet. Zusammenfassend und zu weiteren Forschungsdesideraten Klaus Herbers: Heilige an den Grenzen „Lateineuropas“, in: Sakralität zwischen Antike und Neuzeit, hg. v. DEms./Berndt Hamm/ Heidrun Stein-Kecks, Stuttgart 2007 (Beiträge zur Hagiographie 6), S. 101-111, hier S. $103-105$. 
Translationsbericht erzählt? Die Muslime hätten demnach die Liparischen Inseln geplündert und dabei auch das Apostelgrab nicht geschont. Darauf hatte ein griechischer Mönch eine Vision ${ }^{45}$, in der ihm aufgetragen wurde, er solle die durch den Angriff zerstreuten Gebeine sammeln. Der Mönch lehnte dies zunächst mit sehr handfesten Argumenten ab: Schließlich habe Bartholomäus vor den Muslimen keinen Schutz gewährt. Der Heilige machte demgegenüber geltend, er habe durchaus für das Inselvolk gebetet, das jedoch durch eigene Schwächen selbst an der Entwicklung schuld sei, und ermahnte den griechischen Mönch erneut, die Gebeine zu sammeln. Als der Mönch erwiderte, er könne kaum alle zerstreuten Knochen finden, antwortete der Heilige, bei Anbruch der Dunkelheit würden diese in der Dunkelheit leuchten. Der griechische Mönch gehorchte, jedoch führt die Erzählung nun die Langobarden ein, die den Leichnam des Apostels abholten. Auf dem Meer wurden sie von Sarazenen angegriffen, aber der Apostel rettete und beschützte sie. Dann wurde Bartolomäus cum magno honore recte ${ }^{46}$ nach Benevent gebracht.

Die Sarazenen stören die Ordnung, aber die Griechen haben dem nichts entgegenzusetzen, so daß die Langobarden im Translationsbericht folgerichtig als die eigentlichen Befrieder der Gegend erscheinen. Selbstredend ist die translatio aus langobardisch-beneventanischer Perspektive geschrieben. Mit Bartholomäus wurde Benevent durch einen Sakralisierungsschub - immerhin besaß man nun einen Apostelleichnam - zunächst zu einem Bollwerk gegen die Muslime, weil die Griechen versagt hatten.

Erst über ein Jahrhundert später entfaltete der Kult weitere Wirkkräfte. Wolfgang Huschner erläutert in diesem Band seine Thesen weiter, inwieweit die Gründung des Erzbistums Benevent $969^{47}$ in den Zusammenhang einer Auseinandersetzung zwischen östlichem und westlichem Kaiserreich gehört, die an dieser Stelle kirchenpolitisch ausgetragen wurde ${ }^{48}$. Weitere Erzbistumsgründungen wie Salerno oder Capua erfolgten fast zeitgleich oder wenig später. Dabei erwuchs aber in Benevent mit einer Aposteltradition auch für Rom bis ins hohe Mittelalter eine Konkurrenz, wie bischöfliche Kopfbedeckung, Ehrenvorrechte $^{49}$, Urkundenwesen ${ }^{50}$ und weiteres belegen. Die Apostelreliquien und

45 ... cuidam Graeco monacho ..., Anastasius Bibliothecarius, ed. Westerbergh (wie Anm. 44) S. 11.

46 Ebd. S. 12.

47 JL 3738; IP 9 S. 54 Nr. 15. RI 2/5 Nr. 459.

48 Siehe unten S. 87-108; vgl. bereits Wolfgang Huschner: Benevent - Capua - Magdeburg - Salerno. Neue Erzbistümer an der Peripherie des lateinischen Europa im 10. Jahrhundert, in: Der Hoftag in Quedlinburg 973. Von den historischen Wurzeln zum Neuen Europa, hg. v. Andreas Ranft, Berlin 2006, S. 37-49, hier S. 43.

49 Bernhard Schimmelpfennig: Die Tiara des Erzbischofs von Benevent, in: Ex ipsis rerum documentis. Beiträge zur Mediävistik. Festschrift für Harald Zimmermann zum 65. Geburtstag, hg.v. Klaus Herbers/Hans Henning Kortüm/Carlo Servatius, Sig- 
die Grabestradition wurden in späterer Zeit zunehmend weniger gegen Byzanz oder die Muslime als vielmehr gegenüber Rom wirksam ${ }^{51}$. Die Möglichkeiten der Päpste, hier zu integrieren, waren gering und verlangten einen langen Atem. Es fällt jedoch auf, daß die Dokumentation zur Erhebung und päpstlichen Bestätigung der verschiedenen Erzbistümer - und das ist vor dem Hintergrund unserer quellenmäßigen Auswahl wichtig - nur zu einem Teil in Form von Papsturkunden überliefert ist: im Falle Capuas durch die historiographische Überlieferung ${ }^{52}$, im Falle Salernos sind es spätere Urkunden, welche die Erhebung 983 erkennen lassen ${ }^{53}$.

\section{1054 oder 1059? Die normannische Entscheidung}

Nicht 1054 in Konstantinopel, sondern viel eher 1059 auf der Synode von Melfi mit den sich daraus ergebenden Folgen sei das Schisma zwischen Ost- und Westkirche begründet worden, dies hat jüngst Axel Bayer noch einmal unterstrichen $^{54}$. Wurde die Glaubensspaltung in Süditalien durch Papsttum und Normannen besiegelt? Das Entstehen der normannischen Herrschaft in Süditalien mischte die Karten noch einmal grundlegend neu, schon Leopold Ranke bemerkte in seiner Weltgeschichte, daß dies mehr als vieles andere die Dekomposition verschiedener Großreiche der damaligen Zeit zur Folge gehabt habe ${ }^{55}$. Dabei blieb die Position der Päpste zunächst ausgrenzend. Die Nor-

maringen 1991, S. 363-371, besonders S. 368 f., zur Grenzsituation Benevents und entsprechenden symbolischen Akten (mit weiterer wichtiger Literatur S. 369 Anm. 40). Vgl. weiterhin DERs.: Ein Bischof dem Papste gleich? Zu den Insignien und Vorrechten des Erzbischofs von Benevent, in: Aus Archiven und Bibliotheken. Festschrift für Raymund Kottje zum 65. Geburtstag, hg. v. Hubert Mordek, Frankfurt am Main 1992 (Freiburger Beiträge zur mittelalterlichen Geschichte 3), S. 391-411; Hans Belting: Studien zum beneventanischen Hof, in: Dumbarton Oaks Papers 16 (1962) S. 141 193, und DERs.: Studien zur beneventanischen Malerei, Wiesbaden 1968 (Forschungen zur Kunstgeschichte und zur christlichen Archäologie 7).

50 Herbert Zielinski: Fra ,charta' e documento pubblico: gli atti dei vescovi della Longobardia meridionale, in: Andenna/Picasso (wie Anm. 44) S. 149-176.

51 Vgl. Herbers: Heilige (wie Anm. 44) S. $104 \mathrm{f}$.

52 JL Bd. 1 S. 471; IP 8 S. 223 Nr. *34; RI 2/5 Nr. 393.

53 IP 8 S. 345 Nr. *10; RI 2/5 Nr. 618.

54 Axel BAyer: Spaltung der Christenheit. Das sogenannte Morgenländische Schisma von 1054 (Beih. AKu 53) 2. unveränderte Auflage, Köln/Weimar 2004, bes. S. 122-124 und zusammenfassend S. 206-208. - Zu Melfi vgl. auch Georg Gresser: Die Synoden und Konzilien in der Zeit des Reformpapsttums in Deutschland und Italien von Leo IX. bis Calixt II. 1049-1123, Paderborn 2006 (Konziliengeschichte, Reihe A), S. 48-51 (beide mit der älteren Literatur).

55 Hierzu wiederum Paul Fridolin KeHR: Die Belehnungen der süditalienischen Normannenfürsten durch die Päpste (1059-1192), Berlin 1934 (AAB, phil.-hist. Kl.), 
mannen waren für die Päpste zunächst nur ein neuer Gegner in einer alten Grundkonstellation, denn es fällt zumindest auf, daß die Berichte über die Kämpfe Leos IX. gegen die Normannen diffuse Begrifflichkeiten verwenden. Meinten Saraceni oder Agareni eigentlich nur Muslime, so wurden dies bald Begriffe für den Gegner schlechthin, jedenfalls heißen die Normannen in manchen päpstlich bestimmten Quellen Agareni ${ }^{56}$.

Mit Nikolaus II. begann jedoch eine neue päpstliche Politik. Jedenfalls begründete dieser Papst im August 1059 ein Lehnsverhältnis mit Robert Guiscard $^{57}$. Hinter dieser neuen Orientierung nach den verschiedenen Auseinandersetzungen mit den in Süditalien expansiv agierenden Normannen standen unter anderem Subdiakon Hildebrand und Abt Desiderius von Montecassino. Der Papst legitimierte in Melfi aber die Herrschaft Robert Guiscards auf Kosten von Byzanz ${ }^{58}$. Zu den langfristigen Konsequenzen weiterer Belehnungen bis hin in staufische Zeit hat wiederum Paul Fridolin Kehr bei der Vorbereitung der IP 8 und 9 mit seinem Beitrag zu den Belehnungen der süditalienischen Normannenfürsten durch die Päpste (1059-1192) den Grund für weitere Interpretationen gelegt ${ }^{59}$. Es ist auffällig, wie sehr er dieses Ereignis als Einschnitt ansah, vieles zuvor - die byzantinische oder die sarazenische Herrschaft sowie die verschiedenen Prinzipate - eher als Vorgeschichte dem unterordnete ${ }^{60}$. Allerdings ist zu unterstreichen, daß das Normannenreich Rogers und der beiden Wilhelme aus verschiedenen ,Staaten' und nicht nur aus den Lehnsgebieten des römischen Stuhls erwachsen ist. Insofern war die Integrationskraft der Päpste durch Lehnsbeziehungen - wie dies ja vielleicht trotz einiger jüngster Zweifel zu

S. 355-368, (Nachdr. in: DERs.: Schriften [wie Anm. 9] Bd. 1 S. 591-641, zu Rankes Urteil S. 592).

56 Vgl. hierzu bereits allgemein Carl Erdmann: Die Entstehung des Kreuzzugsgedankens, Stuttgart 1935 (Forschungen zur Kirchen- und Geistesgeschichte 6), S. 110 . - Die Verwendung unter Leo IX. war sicher kein Einzelfall, denn schon im 10. Jahrhundert kritisierte Ekkehard IV. von St. Gallen den diffusen Gebrauch. Er wandte sich dagegen, die Ungarn als Agarener zu bezeichnen: Qui autem Ungros Agarenos putant, longa via errant, Ekkehardus, Casus Sancti Galli, hg. und übersetzt von Hans F. Haefele, Darmstadt 1980 (AusgQ 10), S. 170 (ich benutze die von Hans F. Haefele verbesserte Ausgabe, vgl. zur Sache dort auch S. 171 Anm. 51).

57 Grundlegend hierzu (mit weiteren Belegen und Literatur): Josef DeÉr: Papsttum und Normannen. Untersuchungen zu ihren lehnsrechtlichen und kirchenpolitischen Beziehungen, Köln/Wien 1972 (Studien und Quellen zur Welt Kaiser Friedrichs 2/1), S. 96 f. und 101-103, Vincenzo D'Alessandro: Fidelitas Normannorum. Note sulla fondazione dello Stato normanno e sui rapporti col papato, in: DERs.: Storiografia e politica nell'Italia normanna, Neapel 1978 (Nuovo Medioevo 3), S. 99-220, bes. S. 134-138.

58 Vgl. die verschieden Regesten IP 8 S. 12 Nr. 15 und 16 mit Hinweis auf die Überlieferungen bei Deusdedit III 285 und im Liber Censuum cap. 163 sowie die in der vorigen Anm. zitierte Literatur.

59 KeHr: Belehnungen (wie Anm. 55).

60 Ebd. S. 593. 
anderen Reichen im Orbis christianus angestrebt wurde - zwar gegeben, konnte aber nur teilweise wirken. Hinsichtlich der Überlieferung fällt wiederum auf, daß Registerfragmente, Sammlungen und Notizen von Deusdedit sowie Albinus und Cencius entscheidend für unsere Kenntnisse der Abläufe von Melfi sind ${ }^{61}$. Die Archive der Herzöge von Apulien und der Fürsten von Capua sind vollständig zugrunde gegangen, zum Archiv der Normannenkönige wird von Hugo Falcandus berichtet, daß es im Jahre 1161 beim Aufstand gegen König Wilhelm I. zum größten Teil vernichtet worden sei ${ }^{62}$. So bleibt auch hier für die Beziehungen zur weltlichen Gewalt kaum urkundliche Überlieferung, nur für die geistlichen Institutionen lassen sich die Empfängerarchive rekonstruieren.

Abgesehen von der politischen Tragweite waren die kirchenpolitischen Konsequenzen zentral, denn nach der sukzessiven Errichtung einer einheitlich römisch-lateinischen Hierarchie - auf der Synode von Melfi (eventuell auch erst etwas später) - wurden die Bischöfe Johannes von Trani und Siponto, die mit Byzanz im Einvernehmen standen, abgesetzt ${ }^{63}$. Damit begann ein Prozeß der Latinisierung der Kirche in Süditalien. Die Verdrängung der Sarazenen in Sizilien, der diejenige in Sardinien und Korsika durch Pisa und Genua hinzuzufügen ist, führte dazu, daß die neue kirchliche Landkarte im Süden Italiens besonders verworren erscheint, weil nur teilweise alte Strukturen wiedererrichtet wurden. In der Folge bildete sich dennoch als Grundtendenz heraus, daß nach dem Vertrag von Benevent $1156^{64}$ die Herrscher weitgehend auch den Klerus kontrollierten und indirekt bestimmten, wie weit der Arm des Papstes überhaupt reichen konnte. Wie gering der Einfluß im Vergleich zu manchen Gebieten sein konnte, ist dem Beitrag von Jochen Johrendt zu entnehmen ${ }^{65}$. Daß allerdings die Gestaltung der Papsturkunden die normannische Kanzlei nach 1156 besonders beeinflußte, ist erwiesen, auch wenn Theo Kölzer den von Kehr postulierten „mächtigen Einfluß der Papsturkunde“ inzwischen auf ein realistisches Maß zurückgestutzt hat ${ }^{66}$.

61 Siehe oben Anm. 57.

62 Vgl. KeHr: Belehnungen (wie Anm. 55) S. 594. Zur Nachricht vgl. auch die Einleitung von Horst EnZENsberger: Einleitung, in: Guillelmi I regis diplomata, Köln/Weimar/ Wien 1996 (Codex diplomaticus regni Siciliae 1/3), S. IX-XVI, hier S. X (mit weiteren Hintergrundsinformationen).

63 IP 9 S. 290 Nr. 2 und S. 235 Nr. 11, vgl. Bayer (wie Anm. 54) S. 123 f. mit Anm. 47 zur Forschungsdiskussion.

64 KeHr: Belehnungen (wie Anm. 55) S. $636 \mathrm{f}$.

65 Unten, S. 183-213, dies freilich auch wegen der Konsequenzen der Stellungnahmen im Schisma von 1130.

66 Theo Kölzer: Der Einfluß der Papsturkunde auf die Urkunden der normannischen Könige Siziliens, in: Papsturkunde und europäisches Urkundenwesen. Studien zu ihrer formalen und rechtlichen Kohärenz vom 11. bis 15. Jahrhundert, hg. v. Peter Herde/ Hermann Jаковs, Köln 1999 (Beih. ADipl. 7), S. 307-317, hier S. 315. 


\section{Ubi est papa, ibi est Roma: Papst Lucius III. 1184 im Großeinsatz}

„Ubi est papa, ibi est Roma“, dieser Satz des 13. Jahrhunderts deutet die Schwierigkeiten an, die zuweilen entstanden, wenn man den Hauptort der Christenheit theoretisch festlegen wollte ${ }^{67}$. Er besitzt auch seinen Hintergrund in langen Abwesenheiten der Päpste des 11. und 12. Jahrhunderts von Rom und in den verschiedenen oft lange amtierenden Gegenpäpsten dieser Zeit. Mein letztes Aperçu geht auf diese Aspekte ein und greift einen weiteren Punkt auf, der indirekt alle regional gegliederten Pontificien betrifft. Die Päpste des ausgehenden 11. und 12. Jahrhundert waren wesentlich weniger in Rom, aber auch nicht nur in dem vielgestaltigen Italien unterwegs. Dabei hatten sich neue Institutionen ausgebildet von den Kardinälen bis zur päpstlichen Kanzlei. Legaten wurden zunehmend entsandt, Delegationsmandate ausgestell $t^{68}$.

Vielfältige Fragen standen an, als Lucius III. im Oktober 1184 mit Friedrich I. in Verona zusammentraf. Es waren dies nicht nur Verhandlungen über die Mathildischen Güter, sondern auch Fragen des Kreuzzugs und weitere Punkte. Der neue Band der Regesten Lucius' III., der im Rahmen der Regesta Imperii erschienen ist ${ }^{69}$, läßt nicht nur gut erkennen, wie sehr Lucius III. die Ergebnisse des Dritten Laterankonzils umgesetzt hat, sondern auch, wie leistungsfähig die päpstliche Kanzlei mit ihrem täglichen Ausstoß an Urkunden geworden war ${ }^{70}$. Seit dem 22. Juni 1184 bis zu seinem Tod am 25. November 1185 amtierte Lucius III. in Verona. Mehr als 800 Urkunden und Deperdita verzeichnen die Regesten sicher für diese $\mathrm{Zeit}^{71}$. Als der Kaiser kam, scheint die Kanzlei auf

67 Michele Maccarrone: Ubi est papa, ibi est Roma, in: Aus Kirche und Reich. Studien zu Theologie, Politik und Recht im Mittelalter. Festschrift für Friedrich Kempf zu seinem 75. Geburtstag und fünfzigjährigen Doktorjubiläum, hg.v. Hubert Mordek, Sigmaringen 1983, S. 371-382; Johannes Laudage: Rom und das Papsttum im frühen 12. Jahrhundert, in: Europa an der Wende vom 11. zum 12. Jahrhundert. Beiträge zu Ehren von Werner Goez, hg. v. Klaus Herbers, Stuttgart 2001, S. 23-53, bes. S. 23 und 53.

68 Ich verzichte auf Einzelnachweise, die bequem den verschiedenen Beiträgen des Sammelbandes Das Papsttum in der Welt des 12. Jahrhunderts, hg.v. Ernst-Dieter HeHL/ Ingrid Heike Ringel/Hubertus Seibert, Stuttgart 2002 (Mittelalterforschungen 6) entnommen werden können, vgl. auch zusammenfassend den Einleitungsbeitrag von Ernst-Dieter Hehl: Das Papsttum in der Welt des 12. Jahrhunderts. Einleitende Bemerkungen zu Anforderungen und Leistungen, in: ebd. S. 9-23, sowie die Schlußbemerkungen von Alfons BeCKer: Das 12. Jahrhundert als Epoche der Papstgeschichte, in: ebd. S. 293-323.

69 RI 4/4/4/1-2; hier geht es vor allem um die zweite Lieferung zu den Jahren $1184-$ 1185 .

70 Vgl. bereits die Tabelle bei Rudolf Hiestand: Die Leistungsfähigkeit der päpstlichen Kanzlei im 12. Jahrhundert mit einem Blick auf den lateinischen Osten, in: Herde/ Jаковs (wie Anm. 66) S. 23.

71 Ohne die undatierten und unsicheren Stücke 1181-1185. 
Hochtouren gearbeitet zu haben, mit statistischen Vergleichen hat Rudolf Hiestand dies vor einigen Jahren deutlich hervorgehoben ${ }^{72}$. Der Aufenthalt in Verona läßt aber einen weiteren Aspekt erkennen: Von den Urkunden für italische Personen und Institutionen gibt es aus dieser Zeit nur ganz wenige, die an Empfänger in Mittel- und Süditalien gerichtet waren ${ }^{73}$. Aus der Perspektive der Urkunden dominierte Norditalien. Der Satz Ubi est papa, ibi est Roma heißt also auch, daß der päpstliche Aufenthaltsort die Gänge der Bittsteller an die Kurie durchaus beeinflussen konnte.

Ein weiteres Problem lassen aber diese Urkunden Lucius' III. in Verona darüber hinaus erkennen: Viele galten Ritterorden und Orden. Die teilweise standardisierten Privilegien und Gnadenbriefe wurden aber zuweilen an den Orden allgemein, bald an ein eigenes Haus oder auch an eine Ordensprovinz oder ,Ordenszunge' gerichtet. Die Archivgeschichte zeigt, daß allgemein adressierte Schriftstücke durchaus im Archiv eines bestimmten Hauses aufbewahrt werden konnten. Auch wenn ein bestimmter Adressat genannt wird und wenn es zum Beispiel in der Adresse heißt in Anglia constitutis bedeutete dies nicht automatisch, daß die Vergünstigungen nur für Templer oder Johanniter in England gedacht waren, sondern vielmehr, daß der englische Ordenszweig dieses Privileg erbeten hatte und dann eine Ausfertigung mit dem üblichen Formular erhielt. Wohin gehören diese Briefe aber dann: zur Italia Pontificia, zum Oriens Pontificius oder wozu sonst? Die Beiträge von Rinaldo Comba, Mario Sensi und Kristjan Toomaspoeg berücksichtigen diese Probleme ${ }^{74}$.

\section{Schlußfolgerungen und Fragen}

Was bieten die Aperçus für unsere Fragestellungen? „Unglaubliches ist uns zu Ohren gekommen", so formulierten nicht nur Päpste des 9., sondern auch des 12. Jahrhunderts, obwohl ich diese Aspekte nicht mehr im einzelnen präsentieren konnte. Die Vielfalt Italiens, so dürfte aber hoffentlich deutlich geworden sein, führte dazu, daß die Bemühungen der Päpste um Anerkennung Roms, um Integration und Einfluß, die wir im ganzen Orbis christianus in Ansätzen seit dem 8./9., entscheidend aber seit dem 11./12. Jahrhundert beobachten können, auch auf Italien im kleinen zutreffen konnten. Waren sie manchmal vielleicht sogar besonders schwierig, wie das normannische Reich nahe legen könnte? Meine Beispiele betrafen stärker den Süden und das frühere Mittelalter, sie

72 Hiestand: Leistungsfähigkeit (wie Anm. 70).

73 Abzulesen an der Konkordanztabelle der neuen Nummern mit denen der Italia pontificia bei RI 4/4/4/2 S. 590-595.

74 Unten, S. 515-555, und 557-591. Der Tagungsbeitrag von Mario Sensi konnte nicht in diesen Band aufgenommen werden. 
haben auch versucht, zur Thematik ,Papsttum und Muslime', die mich schon seit langer Zeit beschäftigt, einen fehlenden Beitrag in unserem Band etwas zu kompensieren ${ }^{75}$. Italien war in der fraglichen Zeit sicher ein kleines vielfältiges Europa mit römisch-lateinischen, griechisch-orthodoxen und muslimischen Traditionen, es war - jedenfalls über lange Zeit - kein geographisch geschlossener, Orbis christianus latinus' im kleinen. Insofern erscheinen gerade hier Aspekte der aktuellen Europa-Debatte in der mediävistischen, aber auch in der aktuellen Diskussion wie in einem Brennglas eingefangen ${ }^{76}$. Inwieweit die Päpste aber aufgrund wechselnder Verhältnisse zu einer einheitlichen Kultur beitrugen, integrierend wirken konnten, steht auf einem anderen Blatt. Außerhalb des Patrimonium Petri versuchten sie dies immer wieder, wie Beispiele zu griechischen Traditionen, muslimischen Bedrohungen und das Normannenbündnis verdeutlichen. In den politischen Zuordnungen, die bei meinen Aperçus stärker im Vordergrund standen, waren Schwankungen deutlich; viel eher könnte vielleicht eine kontinuierlich steigende Einflußnahme für die hier weniger beachtete kirchliche Organisation in Liturgie, Verfahrensweisen und anderem gelten. Vielleicht könnte als eine Arbeitshypothese jedoch die Frage formuliert werden, ob der Anteil Italiens an den zahlreichen verschiedenen Kulturen die Vereinheitlichung nach päpstlichem Vorbild gegenüber anderen Ländern des Orbis christianus in unserem Zeitraum eher erschwert hat. Oder anders: Ab welchem Zeitpunkt und in welchen Konstellationen ergaben sich Möglichkeiten, päpstlichen Einfluß geltend zu machen, aber auch Normen und Vorbilder mit Erfolgschancen zu implementieren? Welche Rolle spielten die empfangenden und fordernden Institutionen und Personen in diesem, push and pull'-Prozeß, wie es die Soziologen ausdrücken würden ${ }^{77}$ ?

Wenn die Versuche zu langfristig erfolgreichen Integrationsprozessen auch in den Urkunden bis zum Investiturstreit kaum deutlich werden, vielmehr der Papst im Sinne Jochen Johrendts in den Urkunden in Italien eher als Mitbischof erscheint $^{78}$, so ergeben sich am Ende dieses Beitrags zwei zentrale Aufgaben für die künftige Arbeit: Wie ist dieses Verhältnis zwischen Papst und italischen

75 Ursprünglich war ein solcher Vortrag vorgesehen; hierzu plane ich eine separate Abhandlung.

76 Siehe hierzu oben Anm. 5.

77 Vgl. dazu Klaus Herbers: Im Dienste der Universalität oder der Zentralisierung? Das Papsttum und die „Peripherien“ im hohen Mittelalter - Schlussbemerkungen und Perspektiven, in: Römisches Zentrum und kirchliche Peripherie. Das universale Papsttum als Bezugspunkt der Kirchen von den Reformpäpsten bis zu Innozenz III., hg. v. Jochen Johrendt/Harald Müller, Berlin/New York 2008 (Neue Abhandlungen der Göttinger Akademie der Wissenschaften 2), S. 323-343. Sowie das von den Herausgebern dieses Bandes initiierte internationale DFG-Netzwerk mit dem Titel „Zentrum und Peripherie? Das universale Papsttum und die europäischen Regionen im Hochmittelalter“.

78 Siehe oben Anm. 6. 
Bischöfen sowie geistlichen Institutionen vor 896 und besonders nach $1046 \mathrm{zu}$ bestimmen, sowie, zweitens, wie kann die nichturkundliche Überlieferung, die ja mehrfach zu Wort kam, das Bild verändern? Hier liegt, so glaube ich, eines der Felder für Nachträge zur Italia, aus meinem Arbeitsbereich hatte ich einige Beispiele genannt. Nicht von ungefähr ist die dritte Sektion unserer Tagung am stärksten besetzt. Basierte das Unternehmen, insbesondere der Italia Pontificia anfangs vor allem auf Dokumentation der urkundlichen Überlieferung, die noch durch Nachträge weiter ergänzt werden kann, wie Tommaso di Carpegna Falconieri und Dieter Girgensohn in ihren Tagungsbeiträgen aussprachen ${ }^{79}$, so hat Kehr nur zögernd - auf Drängen Rudolf Smends - auch die Briefe und zuweilen - aber nicht immer - epigraphische Zeugnisse hinzugefügt, worauf Sebastian Scholz eingeht ${ }^{80}$. Erst bei der Arbeit weitete sich die Berücksichtigung der nichturkundlichen Überlieferung kontinuierlich aus. War es im Falle des von mir genannten Schreibens an Abt Honoratus die kanonistische Überlieferung, wohl unter Nutzung päpstlicher Register, über deren Aussagekraft und quellenkritische Schwierigkeiten Lotte Kéry und Rudolf Schieffer Auskunft geben $^{81}$, so zeigten die Fälle Benevents, wie wichtig hagiographische Berichte, die Giulia Barone vorstellt ${ }^{82}$, oder verschriftlichte Erzählungen über den Erwerb von Urkunden werden können, wie Dietrich Lohrmann verdeutlicht ${ }^{83}$. Insgesamt bedingte der vergleichsweise frühe Abschluß der Italia Pontificia auch restriktive Aufnahmekriterien. Welche Bedeutung dennoch Kardinals- und Legatenurkunden haben können, unterstreicht Werner Maleczek ${ }^{84}$. Ob dann die Suche bis zu den literarischen Produktionen der Neuzeit über die Gefangenenfreikäufe bei den Sarazenen führen muß, und wie weit sie überhaupt führen kann, muß zunächst offen bleiben.

Das Papsttum und die, vielen' Italien: Die Tagung und der hier vorgelegte Band führen uns in diese verschiedenen Welten, ihr Anlaß bietet zahlreiche Möglichkeiten, stolz auf das ,Paradestück' des Unternehmens zu blicken. Die Vielfalt des Werkes und Italiens erlauben aber zugleich Anfragen und Anregungen für die Zukunft. Gerade vor dem Hintergrund der Vor- und Nachteile räumlicher Gliederungsprinzipien sei die Frage gestellt, wie sehr es an der Zeit sein könnte, chronologische und regionale Verzeichnisse zu kombinieren, um

79 Unten, S 389-401 und 215-257.

80 Unten, S 373-388.

81 Unten, S 275-297 und 261-273.

82 Unten, S 299-309.

83 Unten, S 311-329.

84 Unten, S 331-372. 
die angedeuteten Probleme einer manchmal künstlichen räumlichen Zuordnung zumindest abzumildern und damit Perspektiven für neue Fragen zu eröffnen ${ }^{85}$.

\section{Riassunto}

Prendendo spunto dall'esempio del secolo IX, l'autore mette in luce la complessità dell'Italia nell'alto e pieno medioevo e si ricollega in questo contesto alle discussioni sulla formazione dell'Europa (latina). In particolar modo esamina l'opera di integrazione e l'azione di disintegrazione del papato. Complessivamente si valutano anche la suddivisione dell'Italia, operata da Fridolin Kehr, in Italia media, Italia superior e Italia inferior, nonché le categorie documentarie poco considerate nel processo di ultimazione dell'Italia Pontificia.

Seguono poi cinque breve riflessioni su questioni che riguardano: 1) Italia e Roma come "unità" concettuale e geografica, 2) l'immagine dell'Italia meridionale come una forma di "Europa variegata" e un "campo di battaglia" per ulteriori conflitti (Bisanzio, il mondo musulmano), 3) rapporti concorrenziali con Roma (ad esempio a Ravenna o a Benevento), 4) la questione normanna del secolo XI, 5) le dimore dei Papi fuori Roma e le consequenze che ne risultano. In questo modo è stato presentato un catalogo di punti che rimandano agli altri contributi del volume.

85 Hierzu sind Initiativen in Erlangen/Göttingen im Gange, die versuchen, Regestentraditionen, die von chronologischen oder von geographisch-institutionellen Gesichtspunkten ausgingen, durch elektronische Aufbereitung zusammenzuführen. 